\title{
Determination of the Total Concentration and Speciation of Metal Ions in River, Estuarine and Seawater Samples
}

\author{
Giancarla Alberti, ${ }^{\dagger}$ Raffaela Biesuz, and Maria Pesavento \\ Dipartimento di Chimica Generale, Università degli Studi di Pavia, via Taramelli, 12-27100 Pavia, Italy
}

\begin{abstract}
Different natural water samples were investigated to determine the total concentration and the distribution of species for $\mathrm{Cu}(\mathrm{II}), \mathrm{Pb}(\mathrm{II}), \mathrm{Al}(\mathrm{III})$ and $\mathrm{U}(\mathrm{VI})$. The proposed method, named resin titration (RT), was developed in our laboratory to investigate the distribution of species for metal ions in complex matrices. It is a competition method, in which a complexing resin competes with natural ligands present in the sample to combine with the metal ions. In the present paper, river, estuarine and seawater samples, collected during a cruise in Adriatic Sea, were investigated. For each sample, two RTs were performed, using different complexing resins: the iminodiacetic Chelex 100 and the carboxylic Amberlite CG50. In this way, it was possible to detect different class of ligands. Satisfactory results have been obtained and are commented on critically. They were summarized by principal component analysis (PCA) and the correlations with physicochemical parameters allowed one to follow the evolution of the metals along the considered transect. It should be pointed out that, according to our findings, the ligands responsible for metal ions complexation are not the major components of the water system, since they form considerably weaker complexes.
\end{abstract}

(Received January 10, 2008; Accepted March 31, 2008; Published December 10, 2008)

\section{Introduction}

The geochemical cycling, the distribution in the environment and the bioavailability of trace metal ions depend on their chemical speciation. Metal species formed by inorganic and organic ligands, at relatively high concentration and with weak complexing properties, determine an important fraction, very reactive, with high mobility and usually high toxicity. However other ligands, able to form very stable complexes, could play a paramount role in metal distributions. They can drastically decrease the free metal concentration by several orders of magnitude, albeit present at concentrations similar to those of the trace metal ions (nM level).

Indeed it has been demonstrated that the detection of these ligands is possible only when extremely low concentrations of metal loadings are employed. ${ }^{1}$ These findings seem consistent with a pattern common to several metal ions: the natural organic matter is more likely to provide continuous binding sites than different discrete ligands. Accordingly, stronger binding sites are utilized at lower metal ion loadings and only progressively weaker sites contribute to metal complexation at higher loadings. ${ }^{1}$

The resin titration (RT) is a method developed in our laboratory $\mathrm{y}^{2,3}$ to investigate the speciation of metal ions at trace level in complex matrices. It is a competition method, in which the competing agent is a complexing resin. It was proved to be particularly useful in the detection and characterization of strong ligand sites, at low concentration, in natural water samples, since the competition strengths of the complexing resins are very high. It is not required to add any metal for the titration, as it is the case of the other electrochemical methods (anodic and

$\dagger$ To whom correspondence should be addressed. E-mail: galberti@unipv.it cathodic stripping voltammetries) usually employed for speciation studies. Moreover, the RT can be used for the speciation of many metal ions simultaneously, and being based on the sorption of the metal ions on the resin and on the determination of the concentrations of sorbed metal ions after elution, many materials that interfere with the final detection are eliminated.

The accuracy of the RT method was verified for the total metal concentration and for the metal complexation with synthetic solutions containing known metals and known ligands. ${ }^{4,5}$ Recently, in an interlaboratory test, ${ }^{6,7}$ a method always based on resin competition was employed to determine the concentration and the conditional constant of a solution of ethylenediaminetetraacetic acid (EDTA), obtaining values in good agreement with those reported in the literature. ${ }^{6}$ Actually in speciation studies, the validation of a method can be achieved either by analyzing known solutions, or by comparing results obtained with other techniques. This is an significant step in development of new methods, especially when the comparison permits one to elucidate advantages and limitations of the proposed techniques. ${ }^{8}$ Moreover, the comparison is fundamental in such cases where certified materials do not exist. ${ }^{9}$ In this framework, we also presented an interlaboratory exercise ${ }^{10}$ to compare our technique with AGNES (absence of gradients and Nernstian equilibrium stripping). ${ }^{11} \quad$ The interest in such comparison was supported by the very different physicochemical natures of the two techniques: RT is based on an ionic exchange resin while AGNES is a voltammetric technique. The results, compared with the calculated values and also with those obtained from ion selective electrodes, demonstrated a good accuracy in the determinations of the free $\mathrm{Pb}(\mathrm{II})$ with the two methods in solutions with known ligands.

In the present investigation, the RT method is proposed for the determination of total concentration and speciation of $\mathrm{Cu}(\mathrm{II})$, $\mathrm{Pb}(\mathrm{II}), \mathrm{Al}(\mathrm{III}), \mathrm{U}(\mathrm{IV})$ in river, estuarine and seawater samples. 


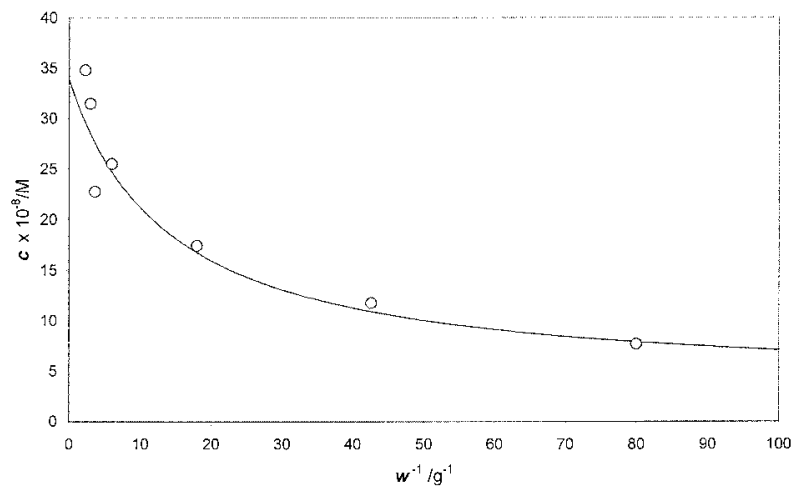

Fig. 1 The resin titration curve for $\mathrm{Al}(\mathrm{III})$ by Chelex 100 in a seawater sample. A volume of $100 \mathrm{ml}$ was employed for each subsample.

These samples were collected during a cruise on the Adriatic Sea in April 2004, in a transect between the mouth of Po River (locality Po di Goro, Italy) and the Central Adriatic, along the plume, direction East-South East. For each sample two RTs were performed on board, immediately after sampling, using different complexing resins: the strong iminodiacetic Chelex 100 and the carboxylic Amberlite CG50, usually a weaker competitor for metal complexation. The usage of two resins with different competition strengths enables one to detect different classes of ligands.

The results are treated by principal component analysis (PCA), considering the variables: $\mathrm{pH}$, temperature, salinity, turbidity, $E_{\mathrm{h}}, \mathrm{O}_{2}$, chlorophyll $a$, the total metal ion content $\left(c_{\text {tot,res }}\right)$ and the concentration of the weakly complexed metal $\left(c_{\mathrm{w}, \mathrm{res}}\right)$, according to the results of RTs as will be shown below.

\section{The basis of the resin titration method}

In the RT method, the resin is the titrant of the metal ion and it is always used in large excess with respect to the metal ions. The procedure is based on the measures of the metals adsorbed on the resin from known volumes of solution $(V$, in $\mathrm{ml})$ when different amounts of resin $(w$, in g) are considered. The RT curve is obtained by plotting the concentration of the metal ion adsorbed on the resin $(c$, in $\mathrm{M}) v s .1 / w$. The titration curve of $\mathrm{Al}$ (III) with Chelex 100, in the case of a seawater sample, is reported in Fig. 1 as an example.

The concentration of sorbed metal ions depends on the species stability in the solution phase and on the sorbing properties of the complexing resin. The stability of the species in solution is measured by the side reaction coefficient of the metal, $\alpha_{\mathrm{M}}$, which is the ratio of total metal ions to free metal ions in solution. ${ }^{12}$ The sorbing properties of the resins are measured by the partition coefficient $K^{*}$, which is the ratio of the metal ion concentration in the resin phase to the free metal ion concentration in solution ${ }^{4,13}$ and which is a measure of the complex stability. $K^{*}$ can be calculated at each particular set of experimental conditions as described in previous papers..$^{413}$

The concentration of metal ions adsorbed at each point of the titration is modelled by the following relationship:

$$
c=c_{\mathrm{w}}+\frac{c_{\mathrm{tot}, \mathrm{res}}-c_{\mathrm{w}}}{1+\frac{\alpha_{\mathrm{M}} V}{K^{*} w}}
$$

$c_{\text {totres }}$ is the total concentration of the metal ion that is sorbed on the resin when $1 / w \rightarrow 0 ; c_{\mathrm{w}}$ represents the fraction of metal

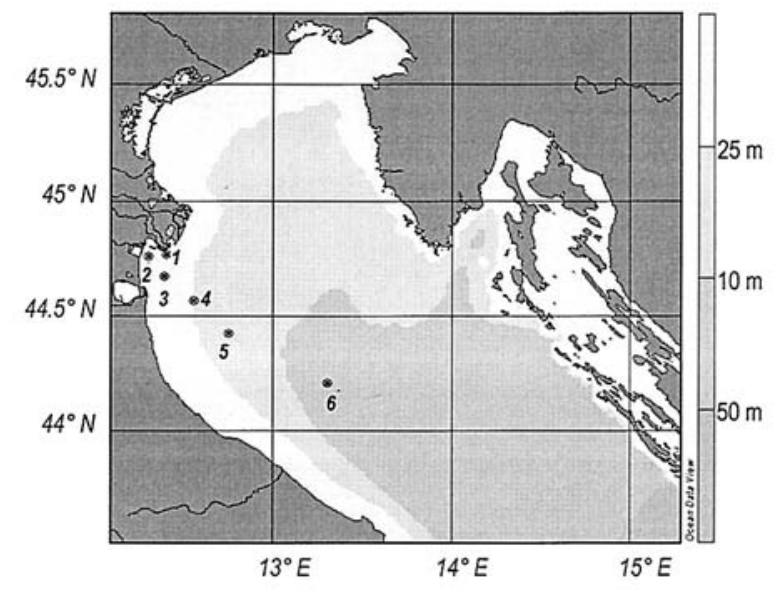

Fig. 2 The map showing the transect of stations studied in the Adriatic Sea, from the mouth of the river Po to the open sea.

which is free or bound in weak complexes quantitatively dissociated by the resin. It can be evaluated directly from the titration curve when a constant concentration of metal ion is adsorbed, i.e. from the points at the lowest amount of resin, where the concentration of adsorbed metal is independent of $1 / w$. The concentration of complexes which compete with the resin is given by the difference $c_{\text {tot,res }}-c_{\mathrm{w}}$. They are able to compete with the resin and their reaction coefficient $\alpha_{\mathrm{M}}$ can be evaluated only if it is in a well defined range of values ("detection window"), which strongly depends on the nature of the resin and on the conditions ( $\mathrm{pH}$, ionic strength). The range is defined by: 3,14

$$
\frac{0.1 K^{*} w}{V}<\alpha_{\mathrm{M}}<\frac{10 K^{*} w}{V}
$$

The central value of the detection window is called the detection strength. In this case the titration curve is similar to that shown in Fig. 1 and $\alpha_{\mathrm{M}}$ can be computed; conversely, if the reaction coefficient $\alpha_{\mathrm{M}}$ is lower than $0.1 K^{*} w / V$, the metal is quantitatively sorbed by the resin at any $1 / w$. It means that $\mathrm{M}$ was either free or combined in complexes with weak ligands completely dissociated by the resin, while if $\alpha_{\mathrm{M}}$ is higher than $10 K^{*} w / V$, the metal ion is so strongly bound to the ligands that it is not sorbed on the resin. The presence of such strongly complexing species cannot be excluded in real samples and for their determination, one must select a resin that is sufficiently strong.

The three parameters $c_{\text {tot,res}}, \alpha_{\mathrm{M}}$ and $c_{\mathrm{w}}$ are evaluated by nonlinear least squares fitting.

\section{Experimental}

\section{Sample collection, treatment and storage}

River, estuarine and seawater samples were collected during a cruise on the Adriatic Sea in April 2004, on the oceanographic boat "G. Dallaporta"-CNR ISMAR Sez. Ancona (Italy), in a transect between the mouth of Po River (locality Po di Goro) and the Central Adriatic, along the plume, direction East-South East. The six sampling points are indicated in Fig. 2: at points 1 and 2 we collected river water, estuarine water was sampled at points 3 and 4 , and at the last two points 5 and 6 , we took seawater samples. Conventional sampling with 10 L PTFE-coated 
Go-Flo bottle (General Oceanics) was used.

Each sample was filtered using membrane filters (pore size, $0.45 \mu \mathrm{m}$ ) immediately after sampling. The $\mathrm{pH}$ of the filtered sample and of the different aliquots after equilibration with the resin was measured by a $\mathrm{pH}$ meter directly on board. A portion of each sample was acidified with nitric acid at $\mathrm{pH}$ around 2 for total metal determination. The RTs were carried out on board immediately after sampling, according to the procedure described below. The separation of the eluting solution $(1 \mathrm{M}$ $\mathrm{HNO}_{3}$ ) from the resin and the final determination of the metals concentration in the eluate were done in the laboratory, approximately three months after sampling. The temperature during transport and storage was $4^{\circ} \mathrm{C}$.

\section{Reagents}

All chemicals were of analytical reagent grade. Solutions were prepared with ultrapure water (Milli-Q). Chelex 100 [CAS 68954-42-7] (Na-form, 100 - 200 mesh) was obtained from BioRad Laboratories and Amberlite CG-50 [CAS 9042-11-9] (weakly acid resin, 100 - 200 mesh) from Sigma-Aldrich. The resins were washed according to the procedure previously described. ${ }^{15}$ This treatment is required to eliminate trace level metal ions that may be present in the resins. The resins were finally converted into the $\mathrm{NH}_{4}{ }^{+}$form by treatment with $1 \mathrm{M}$ high purity ammonia. The capacity of the resins (mmol of active groups per g of dry resin) and the amount of water sorbed under different conditions were determined as previously described. ${ }^{4,16}$

\section{Apparatus}

Ancillary in-situ measurements of $\mathrm{pH}$, temperature, redox potential $\left(E_{\mathrm{h}}\right)$, salinity, oxygen, turbidity and chlorophyll $a$, were made by a CTD sensor (Ocean Seven 316 CTD-Idronaut). CTD data are reported in Table 1 for each sampling point, see columns 1 to 8 .

A portable pH-meter (Model 290A, Orion) and a MettlerToledo (InLab 412) combined glass electrode were used for the $\mathrm{pH}$ determinations on board; the electrode was standardized in $\mathrm{H}^{+}$concentration as previously reported ${ }^{15}$ and used for $\mathrm{pH}$ measurements after filtration and on the different aliquots after RT equilibration.

Conventional sampling with 10 L, PTFE-coated Go-Flo bottle (General Oceanics) was used. Samples were pressure filtrated through an acid-washed membrane filter (mixed esters; $d, 47$ $\mathrm{mm}$; pore size, $0.45 \mu \mathrm{m}$ ( $\mathrm{S} \& \mathrm{~S} 401612)$ ), held in a Nalgene Sulfoflo (300-4100), polysulfone, capacity upper $(500 \mathrm{ml})$, receiver $(1000 \mathrm{ml})$, pressure filtration unit, and using a vacuum pump (vacuum/pressure station Model N. 400-3902-230V AC, Barnant Company).

All the operations on board were made in a laminar flow hood. The bottles for the RTs were prepared in the laboratory before the cruise, placing in each bottle an exactly weighed quantity of dry resin in $\mathrm{NH}_{4}{ }^{+}$form.

An ICP (PerkinElmer SCIEX ELAN 6000 ICP-MS Instrument) was used for the total metal determinations, according to the standard methods recommended by the instrument manufacturer.

\section{Other materials}

Containers, micropipettes, tips and other laboratory materials were made of polyethylene or polystyrene. All the devices were cleaned as previously described. ${ }^{14}$

\section{Procedure for the resin titration $(R T)$}

The titration was carried out by a batch procedure, varying the amount of dry resin $(w)$. Six or seven portions of the same sample $V=100 \mathrm{ml}$, were equilibrated in the RT bottles, previously prepared as reported above, with different amounts of resin (from 0.02 to $0.4 \mathrm{~g}$ of dry resin). The contact time was $24 \mathrm{~h}$ on a shaking plate. A long equilibration is useful when complexes with unknown dissociation rate are present in the sample. If metal species do not dissociate within $24 \mathrm{~h}$, they are considered inert to the method.

After equilibration, the final $\mathrm{pH}$ was measured in each bottle and no attempt was made to keep it at a fixed value. The solution phase was separated by suction and the metal ions were eluted from the resin with $10 \mathrm{ml} 1 \mathrm{M}$ nitric acid by placing it directly into the RT bottles.

The first and most important part of the RT procedure was performed on board, i.e. the sorption of metal ions on the resin and the elution with $1 \mathrm{M} \mathrm{HNO}_{3}$. Then each bottle was stored at $4^{\circ} \mathrm{C}$ for the subsequent determination of metals concentration in the eluting solution, which was done after $c a$. three months in the laboratory. Before analysis these samples were equilibrated at room temperature under a clean hood for $1 \mathrm{~h}$.

\section{Multivariate statistical data analysis}

Principal component analysis (PCA) has been applied to the matrix of the data set obtained from surface samples collected during the Adriatic campaign, with the aims to summarize in a synthetic way the state of the system and to describe for the investigated metals their distributions from the Po mouth to the open sea. The algorithm NIPALS ${ }^{17}$ was applied to the autoscaled matrix of Table 1 .

\section{Results and Discussion}

The water samples, obtained as reported above in the experimental section, were examined by the resin titration method (RT) using Chelex 100 to investigate the presence of strong metal complexes with ligands at low concentration, similar to those found in other seawater samples. ${ }^{14,18}$ The results are reported in Table 1 for each metal as $c_{\mathrm{tot}, \mathrm{Che}}$ and $c_{\mathrm{w}, \mathrm{Che}}$. The values of the competition strengths $\left(\log K^{*} w / V\right)$ are also reported in italics, for each metal and for each RT. The titration with a resin usually having a different detection window, such as Amberlite CG-50, was also carried out for U(VI), $\mathrm{Pb}(\mathrm{II})$ and $\mathrm{Cu}(\mathrm{II})$ in the attempt to examine different competition strengths, so to detect different ligands if possible. The titrations with the two resins were carried out independently. The results of RTs with Amberlite CG 50 are also reported in Table 1, as $c_{\text {tot,Amb }}$ and $c_{\mathrm{w}, \mathrm{Amb}}$ with the related $\log K^{*} w / V$ values in italic.

\section{The total metal concentrations}

The $c_{\text {tot,Che }}$ for $\mathrm{Al}(\mathrm{III}), \mathrm{Pb}$ (II) and $\mathrm{Cu}(\mathrm{II})$ are considered the total dissolved metals. In principle, species with $\alpha_{\mathrm{M}(\mathrm{L})}>$ $10 K^{*}{ }_{\mathrm{Che}} w / V$ cannot be detected, but in a previous investigation on a certified seawater sample CASS 3 at $\mathrm{pH} \sim 5$ adjusted with suprapure solid $\mathrm{Na}_{2} \mathrm{CO}_{3},{ }^{18}$ it has been demonstrated that $c_{\text {tot,Che }}$ corresponds to the total concentration for $\mathrm{Cu}(\mathrm{II}), \mathrm{Cd}(\mathrm{II}), \mathrm{Ni}(\mathrm{II})$ and $\mathrm{Mn}(\mathrm{II})$, while not using Amberlite CG-50. ${ }^{14}$ In the case of $\mathrm{U}(\mathrm{VI})$, Amberlite CG- $50^{19}$ is the strongest sorbent; accordingly, the $c_{\text {tot,Amb }}$ value corresponds to the total dissolved U(VI) concentration in the water samples examined. Since the analytic method employed for metals determinations in this case permits the direct measure of the total metal contents, the analysis on an acidified portion of the original sample was performed and the total metal concentrations were compared with total metal contents obtained from RT with Chelex 100 from $\mathrm{Al}(\mathrm{III}), \mathrm{Cu}(\mathrm{II})$, $\mathrm{Pb}(\mathrm{II})$, with Amberlite CG-50 for U(IV) (Eq. (1)). The results 
Table 1 The data set of the water samples analyzed in the present investigation

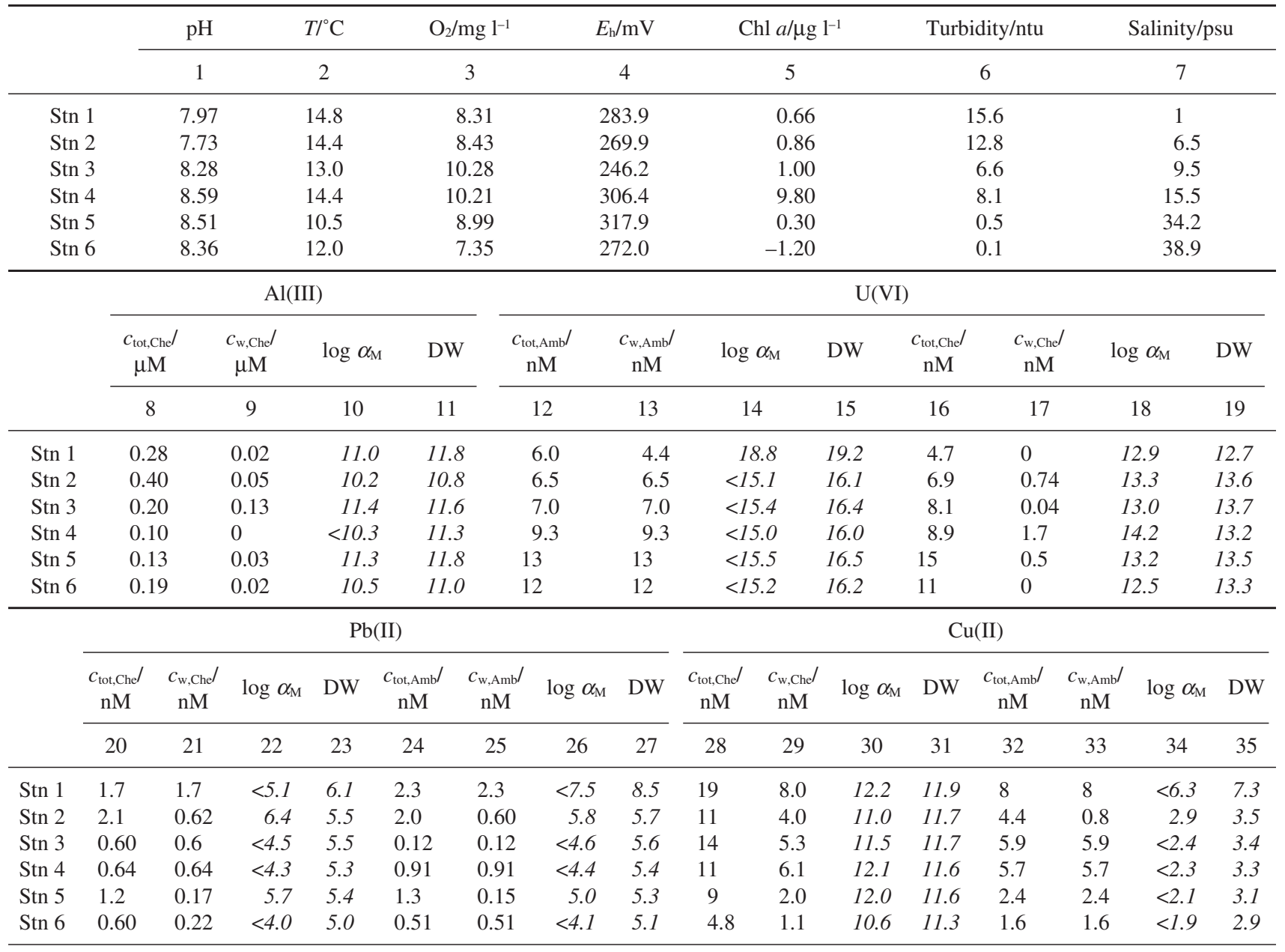

The data reported in column 1 to 7 are the parameters, while the results of RTs are reported for each metal in the following co lumns. The values of $\log \alpha_{\mathrm{M}}$ and of the competition strengths $\left(\mathrm{DW}=\log \left(K^{*} w / V\right)\right.$ are in italics indicating that those columns were not considered further in the PCA.

are reported in Table 2. An intercept not significantly different from zero and a slope not significantly different from 1, at $95 \%$ of confidence level, were always obtained. In Fig. 3 the comparison is summarized in a logarithm graph where the results of the considered metals are reported with circles.

In the case of U(IV) it should be underlined that Amberlite CG-50 is so strong as to give almost always a quantitative sorption ( $c_{\mathrm{tot}, \mathrm{Amb}}$ is equal to $c_{\mathrm{w}, \mathrm{Amb}}$, see Table 1 columns 11 and 12), while Chelex 100 permits a good evaluation of the total concentration, but it competes with the complexes, as could be seen from the results of the titration curves $\left(c_{\text {tot,Che }}\right.$ differs from $c_{\mathrm{w}, \mathrm{Che}}$ ). More consistent data about the total metal content can consequently be obtained, since the $c_{\text {tot,Che }}$ values can be compared with the total concentrations.

In the case of $\mathrm{Pb}(\mathrm{II})$, the properties of the two resins are so $\operatorname{similar}^{20}$ (see columns 19 and 22) that analogous results were expected, indeed the total metal concentrations obtained from RTs with the two resins do not differ.

The $c_{\text {tot }}$ obtained with Chelex 100 in the case of U(IV) and with Amberlite CG 50 for $\mathrm{Pb}$ (II) are also reported in Fig. 3 with triangles to assess the previous statements.

\section{The species distributions}

The information achieved from RTs about species distribution is commented on below.
It should be notice that, in principle it will be possible from $\alpha_{\mathrm{M}}$ values to estimate $K_{\mathrm{i}}^{\prime}$ (the conditional stability constant) if strong simplifications are adopted so that $\alpha_{\mathrm{M}} \cong K_{\mathrm{i}}^{\prime} \cdot c_{\mathrm{L}}$, where $c_{\mathrm{L}}$ is the total ligand concentration. Actually these simplifications are the same adopted in electrochemical studies and indeed we used this simplification in the past $\mathrm{t}^{2,7,13}$ but in the present investigation we prefer this simplest approach (the estimate of the strength of complexation) so that comparison among different metals are easier.

In the case of $\mathrm{Cu}(\mathrm{II})$, the competition strengths of the two sorbents differ so much (see columns 25 and 28) that the ligand sites which compete with Chelex 100 are not the same as those involved in the complexes dissociated by Amberlite CG 50. The first ones are about half the concentration of total copper in the river water samples and they became almost $3 / 4$ decreasing the total amount of metal when seawater samples are considered, more or less exhibiting the same complexation strengths and with a $12.2<\log \alpha_{\mathrm{M}}<10.6$. The weak copper detected with Chelex 100 is the only fraction that it is possible to determine with Amberlite CG 50 (because of its low detection strength). Moreover, it was found that this fraction was always quantitatively sorbed on Amberlite CG 50 and it consists of complexes with rather weak sites or ligands or of free copper itself, since $\log \alpha_{\mathrm{M}}<2$.

Less information about speciation were achieved for the other 
Table 2 The total metal concentrations directly measured compared with those obtained from RTs

\begin{tabular}{|c|c|c|c|c|c|c|c|c|c|c|}
\hline & \multicolumn{2}{|c|}{$\mathrm{Al}(\mathrm{III})$} & \multicolumn{3}{|c|}{$\mathrm{U}(\mathrm{VI})$} & \multicolumn{3}{|c|}{$\mathrm{Pb}(\mathrm{II})$} & \multicolumn{2}{|c|}{$\mathrm{Cu}(\mathrm{II})$} \\
\hline & $c_{\mathrm{tot}} / \mu \mathrm{M}$ & $c_{\text {tot,Che }} / \mu \mathrm{M}$ & $c_{\text {tot }} / \mathrm{nM}$ & $c_{\text {tot, Amb }} / \mathrm{nM}$ & $c_{\text {tot, } \mathrm{Che}} / \mathrm{nM}$ & $c_{\mathrm{tot}} / \mathrm{nM}$ & $c_{\text {tot,Che }} / \mathrm{nM}$ & $c_{\mathrm{tot}, \mathrm{Amb}} / \mathrm{nM}$ & $c_{\mathrm{tot}} / \mathrm{nM}$ & $c_{\mathrm{tot}, \mathrm{Che}} / \mathrm{nM}$ \\
\hline Stn 1 & $0.28(3)$ & $0.28(7)$ & $6.0(2)$ & $6.0(2)$ & $4.7(2)$ & $2.0(7)$ & $1.7(2)$ & $2.3(2)$ & $22(3)$ & 19(3) \\
\hline $\operatorname{Stn} 2$ & $0.41(5)$ & 0.4 (1) & $6.2(4)$ & $6.5(1)$ & $6.9(9)$ & $2.3(3)$ & $2.1(5)$ & $2(1)$ & $16(4)$ & 11(1) \\
\hline Stn 3 & $0.26(3)$ & $0.2(1)$ & $7.0(8)$ & $7(1)$ & $8.1(7)$ & $1.1(3)$ & $0.60(2)$ & $0.9(1)$ & $15(2)$ & $14(4)$ \\
\hline Stn 4 & $0.10(1)$ & $0.10(1)$ & $8.4(1)$ & $9.3(7)$ & $8.9(2)$ & $0.8(1)$ & 0.64 & $0.6(1)$ & $11(1)$ & 11(2) \\
\hline Stn 5 & $0.12(1)$ & $0.13(4)$ & $15.3(4)$ & $13(1)$ & $15(2)$ & $1.3(1)$ & $1.2(3)$ & $1.3(3)$ & $9(1)$ & $9(3)$ \\
\hline Stn 6 & $0.16(2)$ & $0.19(5)$ & $12.3(5)$ & $11.6(6)$ & $11(2)$ & $0.6(1)$ & $0.6(1)$ & $0.5(1)$ & $4.4(4)$ & $4.8(3)$ \\
\hline
\end{tabular}

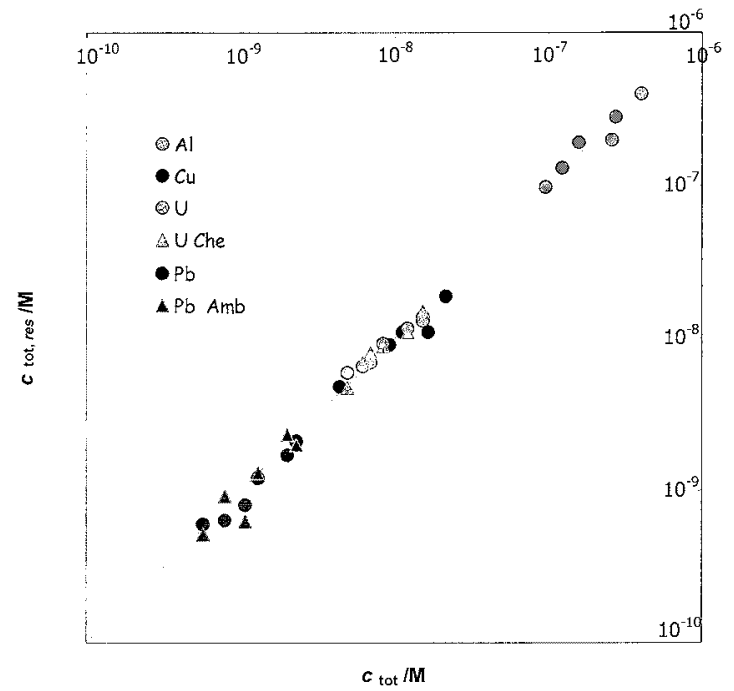

Fig. 3 The comparison between the total metals content directly measured and the total metal determination obtained from the RTs, in a logarithmic scale. With circles the $c_{\text {tot }}$ values obtained from RT with the strongest resin (Chelex 100 for $\mathrm{Cu}(\mathrm{II}), \mathrm{Pb}$ (II) and $\mathrm{Al}(\mathrm{III})$, with Amberlite CG 50 for $\mathrm{U}(\mathrm{IV}))$ are reported, while with triangles the $c_{\text {tot }}$ values obtained with Chelex 100 for $\mathrm{U}(\mathrm{IV})$ and for $\mathrm{Pb}$ (II) with Amberlite CG 50 are reported.

metals. For Al(III) it was not possible to determine in the RTs with Amberlite CG 50, while from the Chelex 100 titrations there was almost always a competition. This means that the complexes responsible for $\mathrm{Al}(\mathrm{III})$ complexation are within the window of the competition strengths, in any case higher than the $\alpha_{\mathrm{M}}$ of the hydrolysis product (at $\mathrm{pH} 7.5, \log \alpha_{\mathrm{hydr}}=7.0$ in seawater and $\log \alpha_{\text {hydr }}=8.4$ in river water).

For $\mathrm{Pb}$ (II) almost overlapping information can be achieved with the two RTs.

The case of U(IV) is rather different since Amberlite CG 50 is so strong as to always dissociate completely the complexes at any $w\left(\log \alpha_{\mathrm{M}}<15.5\right)$. On the other hand Chelex 100 competes with the ligands giving information about their binding strength $\left(14.2<\log \alpha_{\mathrm{M}}<12.5\right)$. In any case Chelex 100 titration allows one to determine the total metal concentration as already commented. It should be noticed that in conditions like those of sample of stn 5, considering hydrolysis products and the contributions of major inorganic ligands (in particular $c_{\mathrm{CO}_{3}}$ around $2 \mathrm{mM}$ ), an estimate of $\log \alpha_{\text {hydr }}=9.58$ was computed in any case several orders of magnitude lower.

\section{Chemiometric analysis}

A detailed chemiometric investigation using PCA is undertaken, in order to summarize in a synthetic way the state of the system and to describe the evolution of metals distribution from the Po mouth to the open sea.

The data set of Table 1 constitutes the matrix of data, where the columns of the detection strengths were omitted, being a property of the resin in that system. The total metal concentrations directly measured were also not considered since their correlation with the total concentrations obtained from RTs was already discussed and considered. Moreover the loadings plot was easily readable without another variable overlapping $c_{\text {tot, Che. }}$.

The values of cumulative variances of the first components are reported in Table 3. Figure 4 shows the scores (a) and the loadings (b) on the plane of the first two eigenvectors (explained variance $74 \%$ ) considered as principal ones.

The first component (explained variance 53\%) expresses the effect of the waters moving from the river (the objects are displaced on the right part of the scores plot) towards the open sea (objects on the left part of scores plot). Indeed the PC1 is dominated by salinity $(-0.285)$, in contrast with turbidity (0.2889) and temperature (0.2579) as seen in Fig. 4b, where the loadings plot of the two first components is reported. Remarkably, all metals and all fractional concentrations decrease with salinity, according to the typical behaviors of heavy metals, in contrast with that of U(VI). It is well known to increase its concentration with salinity. This also happens for its strongest fractions.

The total concentration detected from RTs with the two resins almost overlap as expected, for different reasons, for U(IV) and $\mathrm{Pb}(\mathrm{II})$.

In the second component, the association of the weakest fractions of all metals with the oxygen and chlorophyll $a$ contents is remarkable (Fig. 4b). Indeed in PCA2, $\mathrm{O}_{2}$ and chlorophyll $a$ have the same positive sign, and within a metal distribution the most positive sign (for $\mathrm{Cu}$ and $\mathrm{Pb}$ ), or at least less negative (for $\mathrm{Al}$ ), is that of the weakest fractions. Oxygen and chlorophyll $a$ both increase along the transect, having their maximum values in the samples 3 and 4 , while they decrease again towards the open sea (Fig. 4a). Once more, the estuary shows a high dynamic chemistry where most of the metals, mainly the weakest fractions, react with the organic matter and transfer to sediments. Indeed, moving from the mouth to the sea, the total concentrations decrease, as expected, and in any case the distribution of species is dominated by the strong fraction, while the weak species are those involved in transferring the metal ions to sediments when river waters mix with the salt waters.

The U(VI) data are consistent with those reported in the literature ${ }^{21}$ reaching in the open sea typical values of around 15 $\mathrm{nM}$ (this could be seen from Figs. 4a and 4b: in PCA1 the coefficients of $U$ have always the same sign of the salinity. It means that increasing the salinity, the $U$ concentrations increase), but even in this case the mobile fraction $\left(U_{w}\right.$ Che $)$ is 
Table 3 (a) The percentage of variance cumulatively explained by the first four eigenvectors (95\% limit at which nipals stops, the first two are considered as principal), (b) the score values of the objects on PC1 and PC2, (c) the loadings of the variables on PC1 and PC2

\begin{tabular}{|c|c|c|c|c|c|c|c|c|}
\hline \multicolumn{3}{|l|}{ (a) } & \multicolumn{2}{|l|}{ Env 1} & \multicolumn{3}{|c|}{ Env 3} & Env 4 \\
\hline \multicolumn{3}{|c|}{ Explained variance, $\%$} & \multicolumn{2}{|l|}{54.27} & 74.451 & \multicolumn{2}{|c|}{87.00} & 95.10 \\
\hline (b) & \multicolumn{2}{|c|}{ Stn 1} & Stn 2 & Stn 3 & \multicolumn{2}{|c|}{ Stn 4} & $\operatorname{Stn} 5$ & Stn 6 \\
\hline PCA1 & \multicolumn{2}{|c|}{4.919} & 1.954 & 0.752 & \multicolumn{2}{|c|}{-0.090} & -3.851 & -3.684 \\
\hline PCA2 & \multicolumn{2}{|c|}{-0.132} & -2.334 & 0.231 & \multicolumn{2}{|c|}{3.690} & -0.019 & -1.436 \\
\hline (c) & $\mathrm{pH}$ & $T$ & $\mathrm{O}_{2}$ & $E_{\mathrm{h}}$ & Chl $a$ & Turbidity & Salinity & \\
\hline PCA1 & -0.200 & 0.258 & 0.038 & -0.097 & 0.036 & 0.289 & -0.285 & \\
\hline \multirow[t]{2}{*}{ PCA2 } & 0.336 & 0.088 & 0.351 & 0.223 & 0.430 & -0.005 & -0.038 & \\
\hline & $\mathrm{Al}_{\text {tot che }}$ & $\mathrm{A} 1_{\mathrm{w} \text { che }}$ & $\mathrm{U}_{\text {tot che }}$ & $\mathrm{U}_{\mathrm{w} \text { che }}$ & $\mathrm{U}_{\text {tot amb }}$ & $\mathrm{U}_{\mathrm{w} \text { amb }}$ & & \\
\hline PCA1 & 0.176 & 0.045 & -0.281 & -0.291 & -0.277 & -0.016 & & \\
\hline \multirow[t]{2}{*}{ PCA2 } & -0.360 & -0.123 & 0.040 & 0.038 & 0.025 & 0.323 & & \\
\hline & $\mathrm{Pb}_{\text {tot che }}$ & $\mathrm{Pb}_{\mathrm{w} \text { che }}$ & $\mathrm{Pb}_{\text {tot amb }}$ & $\mathrm{Pb}_{\mathrm{w} \text { amb }}$ & $\mathrm{Cu}_{\text {tot che }}$ & $\mathrm{Cu}_{\mathrm{w} \text { che }}$ & $\mathrm{Cu}_{\text {tot amb }}$ & $\mathrm{Cu}_{\mathrm{w} \text { amb }}$ \\
\hline PCA1 & 0.173 & 0.270 & 0.213 & 0.218 & 0.265 & 0.264 & 0.271 & 0.182 \\
\hline PCA2 & -0.220 & 0.095 & -0.207 & 0.055 & 0.085 & 0.214 & 0.173 & 0.282 \\
\hline
\end{tabular}

(a)

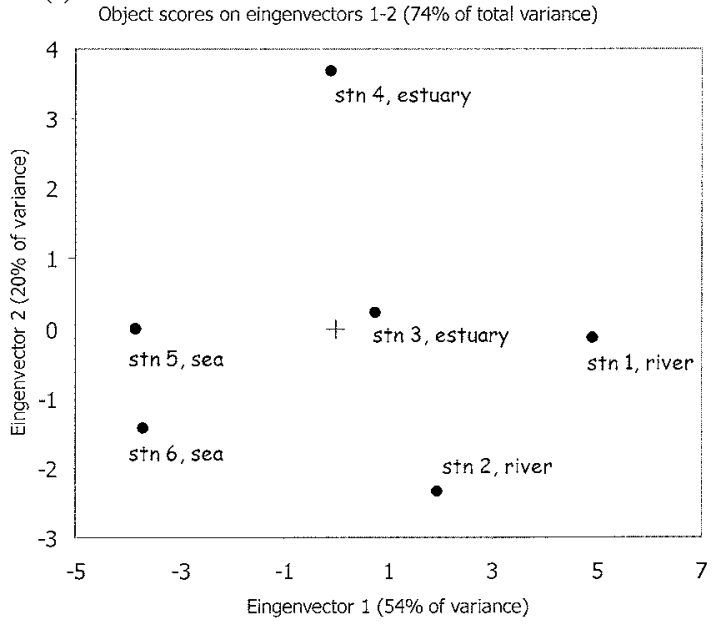

(b)

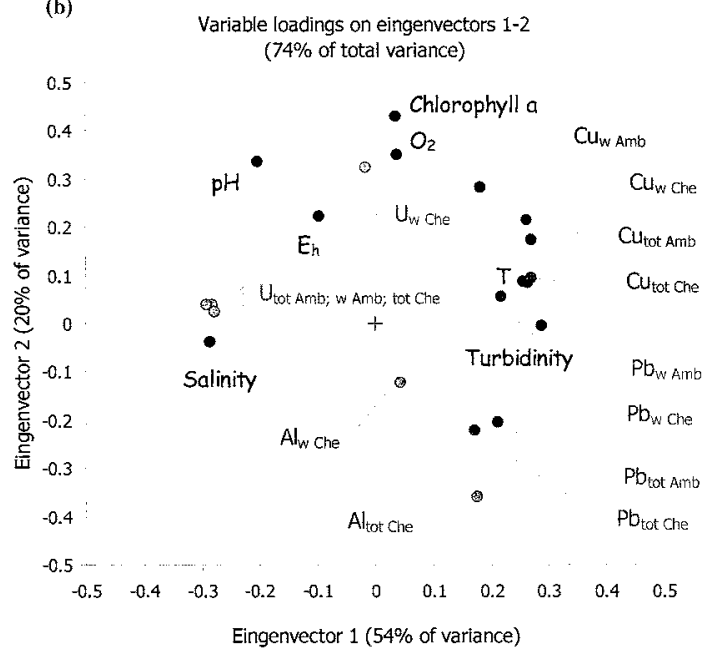

Fig. 4 Principal component analysis results on data sets of Table 1. (a) The scores plot on the two first components, (b) the loadings plot on the two first components. Legend: $c_{\text {totres }}$, total concentration obtained by RT; $c_{\mathrm{w}, \text { res }}$, concentration of the free or weakly complexed metal. The subscript "res" refers to the resin; Chelex 100, "Che"; Amberlite CG50, "Amb".

correlated with the estuary waters, indicating that probably the complexation of $\mathrm{UO}_{2}{ }^{2+}$ changes from the river water to the sea.

The $\mathrm{pH}$ also increases with salinity as expected and this seems in some way associated with chlorophyll $a$ and $\mathrm{O}_{2}$ values; additionally the association of $\mathrm{O}_{2}$ content with the parameter $E_{\mathrm{h}}$ is likely. ${ }^{22}$

\section{Conclusions}

The RT method was used to determine the distribution of the species in a strongly dynamic system, the Po estuary. Two resins were tested, in principle with different sorbing properties. The data set, together with the physicochemical parameters, are summarized by PCA. The model proposed through PCA is indeed based only on a few samples, more samples would be necessary to increase the consistency of the system, nevertheless the exercise once more points out that the powerful tool may be used to follow the evolution of a metal ion in very dynamic conditions and, on the other hand, the RTs results confirm that these resins can contribute, as useful sensors, to understand the complexation of the metals.

The drawbacks of RTs are the need to evaluate $K^{*}$ and the time required for equilibration. The behavior of these two resins was deeply investigated and it was possible to calculate $K^{*}$ for any solution, for several other metal ions, considering the $\mathrm{pH}$ the ionic strength and the presence of high concentration of alkaline earth metals, as in the case of seawater samples. The calculations through which $K^{*}$ is computed were omitted here, but they are reported in several papers or they could be requested from the authors. The equilibration time is not different from that recently adopted in other competitive methods where 
ligands are added to the solution..$^{23,24}$

On the other hand, the advantages consist in the determination of several metal ions, in principle all metal ions that interact with the resins; the possibility of determining the total metal concentrations and the in situ determinations. In particular, concerning this last aspect, we will focus on miniaturizing the RT so that smaller volume and amounts of resins will be needed. Membranes with properties equal to that of Chelex 100 were already tested: their usage makes more easy the manipulation of the samples. For in situ measurements, efforts are also devoted to consider the original temperature of the sample and to perform the equilibration at the same temperature. This effect was not dramatic for the samples considered here but it could be of interest when natural water sampled in extreme conditions is considered.

\section{Acknowledgements}

We want to thank Prof. Giuseppe Scarponi for his invitation on the cruise on the oceanographic boat "G. Dallaporta"-CNR ISMAR Sez. Ancona (Italy). We thank the captain and the crew for their assistance and helpfulness during the on-board-lab operations. This work was financially supported by the PRNA (National Program of the Antarctic Research), grant PEA 2006 (Prog. 9.01) of the Italian Ministry of University and Scientific Research (MIUR).

\section{References}

1. R. M. Town and M. Filella, Sci. Total Environ., 2002, 300, 143.

2. R. Biesuz, G. Alberti, G. D’Agostino, E. Magi, and M. Pesavento, Mar. Chem., 2006, 101, 180.

3. G. Alberti, R. Biesuz, G. D’Agostino, G. Scarponi, and M. Pesavento, Talanta, 2007, 71, 706.

4. M. Pesavento and R. Biesuz, Anal. Chem., 1995, 67, 3558.

5. G. Alberti, R. Biesuz, and M. Pesavento, React. Funct. Polym., 2007, 67, 1083.

6. E. van Veen, S. Comber, and M. Gardner, J. Environ. Monit., 2002, 4, 116.
7. M. Pesavento, R. Biesuz, G. Alberti, A. Profumo, and G. D'Agostino, Chem. Speciation Bioavailability, 2004, 16(1/2), 35 .

8. E. R Unsworth, K. W. Warnken, H. Zhang, W. Davison, F. Black, J. Buffle, J. Cao, R. Cleven, J. Galceran, P. Gunkel, E. Kalis, D. Kistler, H. P. Van Leeuwen, M. Martin, S. Noel, Y. Nur, N. Odzak, J. Puy, W. van Riemsdijk, L. Sigg, E. Temminghoff, M. L. Tercier-Waeber, S. Toepperwien, R. M. Town, L. P. Weng, and H. B. Xue, Environ. Sci. Technol., 2006, 40, 1942.

9. H. P. van Leeuwen, R. M. Town, J. Buffle, R. Cleven, W. Davison, J. Puy, W. H. van Riemsdijk, and L. Sigg, Environ. Sci. Technol., 2005, 39, 8545.

10. G. Alberti, R. Biesuz, C. Huidobro, E. Companys, J. Puy, and J. Galceran, Anal. Chim. Acta, 2007, 599, 41.

11. J. Galceran, E. Companys, J. Puy, J. Cecília, and J. L. Garcés, J. Electroanal. Chem., 2004, 566, 95.

12. A. Ringbom and E. Still, Anal. Chim. Acta, 1972, 59, 143.

13. M. Pesavento, R. Biesuz, F. D. Riva, and G. Alberti, Polyhedron, 2002, 21, 1343.

14. M. Pesavento, R. Biesuz, C. Gnecco, and E. Magi, Anal. Chim. Acta, 2001, 449, 23.

15. M. Pesavento, R. Biesuz, M. Gallorini, and A. Profumo, Anal. Chem., 1993, 65, 2522.

16. M. Pesavento, R. Biesuz, and J. L. Cortina, Anal. Chim. Acta, 1994, 298, 225.

17. R. Brereton, "Chemometrics. Data Analysis for the Laboratory and Chemical Plant", 2003, John Wiley \& Sons Ltd., Chichester.

18. M. Pesavento, R. Biesuz, F. Baffi, and C. Gnecco, Anal. Chim. Acta, 1999, 401, 265.

19. M. Pesavento, R. Biesuz, G. Alberti, and M. Sturini, Anal. Bioanal. Chem., 2003, 376, 1023.

20. R. Biesuz, G. Alberti, and M. Pesavento, J. Solution Chem., 2008, 37, 527.

21. H. Windom, R. Smith, F. Niencheski, and C. Alexander, Mar. Chem., 2000, 68, 307.

22. W. Stumm and J. J. Morgan, "Aquatic Chemistry", 1996, Wiley-Interscience, New York.

23. C. M. G. van den Berg, Anal. Chem., 2006, 78, 156.

24. J. Nuester and C. M. G. van den Berg, Anal. Chem., 2005, $77,11$. 